\title{
Research on Quanzhou Nanyin Culture Promotion Strategy Based on "Maritime Silk Road" Strategy
}

\author{
Linjing Chen \\ Tan Siu Lin Business School \\ Quanzhou Normal University \\ Quanzhou, China
}

\begin{abstract}
Nanyin is a unique cultural resource of Quanzhou, and its protection and inheritance development is of great significance. Based on the current development of Nanyin culture, this paper analyzes the problems existing in the current Nanyin culture communication, and proposes corresponding development strategies based on the current "Maritime Silk Road" development strategy, trying to better inherit and develop Nanyin culture and promote the Nanyin culture at home and abroad so as to provide suggestions for promoting the transmission of China's excellent traditional culture.
\end{abstract}

Keywords-"Maritime Silk Road" strategy; Quanzhou Nanyin culture; promotion; Internet plus

\section{INTRODUCTION}

Nanyin is one of the oldest surviving music species in China, with its distinctive historical culture and humanistic charm. It is circulated in Quanzhou and south Fujian area, also known as "Quanzhou Nanyin". On September 30, 2009, Nanyin applied for a successful legacy and was included in the "Representative List of Intangible Cultural Heritage of Humanity". Although its inheritance and protection have received wide attention from the whole society, its road to communication still faces many difficulties. Nanyin is both a gem of Chinese art and a valuable legacy of the world. Especially in the context of the "One Belt and One Road" strategy, the promotion of Nanyin culture has important practical significance for the inheritance of "Maritime Silk Road" culture.

\section{The Status QuO OF QUANZHOU NANYIN CUltuRE} PROMOTION

\section{A. Overview of Nanyin Culture}

Nanyin originated from Quanzhou, Fujian Province, also known as "Nanqu" and "Nanguan". It is an ancient art that sings in Minnan dialect and has a strong local culture. It brings together the essence of gagaku of the Central Plains since the Tang Dynasty. It draws on the characteristics of Yuan verse and Geyang tune, and gradually merges with the folk music of Minnan. On October 1, 2009, Nanyin was officially listed by UNESCO as an intangible cultural heritage of humanity. Traditional culture still plays an irreplaceable role in the development of Chinese society. It reflects the untiring spirit of pursuing self-improvement and follows the spirit of the times. Therefore, it is of great significance for the protection and inheritance of Quanzhou Nanyin.

\section{B. Current Status of Nanyin Culture Promotion}

In recent years, the promotion of Nanyin culture has received many attention and joint efforts. The government, local schools and the society have made many efforts for the inheritance and promotion of Nanyin culture. At the same time, Nanyin cultural workers innovate the Nanyin cultural works in order to meet the needs of the new audience, forming a new scene of Nanyin.

1) Government support: The Quanzhou government department started the protection and development of Nanyin very early. Since 2002, the municipal government has begun to report to the UNESCO on the intangible cultural heritage of Nanyin, and has spent eight years of capacity. In addition, it adopted the school education to promote the development of Nanyin, strengthened the propaganda of Nanyin, and vigorously promoted the collection of Nanyin resources, which effectively promoted the inheritance and promotion of it. In order to further cooperate with the teaching work, the Quanzhou Municipal Bureau of Culture cooperated with the Municipal Education Bureau to compile the Nanyin Supplementary Textbook for primary and secondary schools.

2) School education: Since the 1990s, many primary and secondary schools in Quanzhou have developed Nanyin's courses, and have held several Nanyin singing competitions in primary and secondary schools. What is worthy of attention is the establishment of the Nanyin undergraduate major. In 2003, the Music and Dance Academy of Quanzhou Normal University established the Nanyin specialty, creatively combining Chinese folk music with undergraduate teaching, using a professional and systematic teaching model and coordinating excellent teaching staff to cultivate high-quality talents from Nanyin specialty, which provides a powerful condition for the inheritance and development of Nanyin.

3) Social participation: The inheritance of the music association is a development model of the Nanyin tradition. 
There are no strict restrictions on the selection of members. Anyone who loves Nanyin and is proficient in Nanyin can join. It is understood that there are more than 200 Nanyin associations in almost every county and city in Quanzhou. Among them, Jinjiang Anhai Yasong Music Association is relatively famous. There are many places worthy of reference in its business development, such as hiring professional Nanyin teachers to improve the performance of the members of the Nanyin performance; actively exploring the market and performing Nanyin performance for free; actively strengthening communication with overseas Nanyin community and so on.

\section{4) Nanyin new image:}

a) Nanyin's new work:

In October 2015, Nanyin's new work "Feng Qiu Huang" premiered at the Liyuan Theatre in Quanzhou and received a good social response. The play attracted many young audiences, indicating that it fits well with the aesthetics of modern young people. The play has innovated Nanyin from the perspective of artistic creation: on the one hand, it shows the role of colleges and universities in inheriting and developing excellent traditional culture; On the one hand, it has actively explored the innovation and development of Nanyin. The creative aspect conforms to the contemporary aesthetic. The stage lighting and the dance beauty are implemented in accordance with international standards, giving the audience a double impact of sight and hearing.

\section{b) Internet plus Nanyin:}

"Internet plus Nanyin" culture brand promotion must first consider the establishment of the official website, and an excellent official website is conducive to enhance the public's trust and recognition of the brand so as to achieve brand promotion and enhance brand value at a lower cost [1]. Fujian Nanyin.com was established in 2007. The logo of the homepage is the image of the petrolic pipa in the Nanyin performance. The webpage contains many categories: Nanyin news, Nanyin music score, Nanyin music library, Nanyin image, Nanyin classroom, Nanyin encyclopedia, Nanyin library, Nanyin gallery. Nanyin association, Nanyin mall, Nanyin's application for the list of world heritage, and also establishes the contribution area and Nanyin forum.

The establishment of Fujian Nanyin official website is of great significance. It can use the Internet for brand promotion to achieve the combination of images, animation and audio. With modern technology, the information is more abundant and the expression forms are more diverse.

\section{THE PROBLEMS IN THE PROMOTION OF QUANZHOU NANYIN CULTURE}

\section{A. Lack of Innovation}

Although Nanyin brings together thousands of years of historical culture and musical charm, Nanyin's development in the current Internet background is not optimistic. With the development of science and technology, the taste of the audience also changes. Cultural consumption tends to be leisure, simple and optimistic. Therefore, Nanyin's singing language, singing content, and media, dance, and lighting techniques of Nanyin need to be reformed to create a new Nanyin that belongs to the Internet age.

\section{B. Lack of Inheritance Talents}

If Nanyin wants to last for a long history, the cultivation of talents is a key link. An excellent inheritor can bring Nanyin out of the country and go to the world. An excellent inheritor not only needs to have a superhard Nanyin playing ability, but also needs to know how to organize Nanyin activities and play a leading role. According to current data, in the Nanyin community in Quanzhou, there are only 28 people who can sing and play Nanyin musical instruments over the age of 65 [2]. Due to the lack of training mechanism for inheritors for a long time, Nanyin lacks talents for inheritance. Therefore, in order to open the Nanyin communication market, talent development is a top priority. It is necessary to cultivate the existing Nanyin talents and develop the reserve talents strength, so as to inject fresh blood for Nanyin's career.

\section{The Degree of Digitization Is Not High}

Digitization means that the existing data is recorded in an orbital format. The user can obtain the required data through the keyword search of the search engine, and they can search the corresponding information directly to their digital information resource system. Nanyin's inheritance mode mainly adopts "spoken-based", which is taught by mouth, not paying great attention to music score, and the music score material is little. As the old generation Nanyin grows old, the classic image also disappears in people's field of vision. According to the survey, there are only 20 audiovisual works of old artists in the 1990s. Theoretical and empirical speeches and characters have no relevant transcripts. Some famous remarks on the tone of the Nanyin masters during their lifetime, such as Chen Dingwu's "Shi Zi Jue", Chen Tianbo's "Five-tones", Lin Wenshu's "Liu Ping" and Zhuang Bulian's pronunciation standard, clearly and smoothly using voice, and holding voice without traces, etc., all of which have no detailed records and explanations [3]. The digitization of these valuable Nanyin materials is not high, which is a great disadvantage to its development.

\section{Lack of Awareness of Network Communication}

Nanyin culture has a history of thousands of years. Although the charm of music and the value of history and culture are intoxicating, its long-term development is a selfentertainment of voiceless singing. Nowadays, the development of the network has penetrated into various cultural fields, and the Internet platform that spans time and space limits provides people with various convenient services. Judging from the current situation, Nanyin's culture communication still stays at the level of word of mouth, and is not in line with the Internet in time. Network application software such as WeChat, microblog, and database are not well established. The lack of awareness of network communication is also one of the factors affecting the spread of Nanyin culture. 
IV. THE PROMOTION STRATEGY OF QUANZHOU NANYIN CUlture Against the "MARITIME Silk RoAD" STRATEGy BACKGROUND

\section{A. The Government Should Increase Support Policies}

At present, the country is committed to promoting cultural prosperity, and governments at all levels attach great importance to local excellent traditional culture. The government's support channels are: firstly, policy support. Guided by national macro-planning, the government should formulate cultural development plans and cultural industry development rules, provide policy support for cultural industry development, and actively protect the cultural industry. To this end, it is necessary to reform the cultural management system, optimize the industrial organization structure, adjust the regional industrial layout, improve the cultural industry legal system, strengthen the cultural market supervision, promote the role of high-tech achievements and cultural industry development, and provide scientific and technological content of cultural product production and cultural services. Policy support for cultural industries should include product innovation, results transformation, technology exchange, and marketing promotion. At the same time, the government must strengthen the construction of public cultural services and create a favorable environment for the development of the Nanyin cultural industry and secondly, financial support: The capital gap in the development of cultural industries is much larger than that of ordinary industries. Although the state has introduced relevant financial support policies, it has encountered bottlenecks due to various reasons and cannot fundamentally solve the financing difficulties of cultural and technological industries. It is recommended that the government set up a special fund for outstanding original works, and at the same time adjust the direction of capital use, and use more funds for the reform of Nanyin and the expansion of Nanyin's communication channels, thus effectively expanding the participation of Nanyin.

\section{B. Multi-channel Folk Promotion}

The strong religious characteristics and numerous folk activities in the southern part of Fujian have largely supported the inheritance and development of Nanyin. It is suggested that people make use of the hard demand of Nanyin performance of the masses in daily life, folk activities, weddings and funerals, to vigorously promote the Nanyin culture, so that the masses have more opportunities to contact Nanyin, subtly forming a feeling for the Nanyin culture and promoting the protection and inheritance of Nanyin. Civil society organizations should exert the power of non-governmental organizations, increase close contact with Nanyin culture in other regions, promote communication, share research results with each other, explore the development path of Nanyin culture, and hand in hand inherit the Nanyin culture. It is necessary to hold Nanyin works creation seminar to explore the innovative Nanyin performance form, and use the "Maritime Silk Road" strategy to promote the Nanyin culture at home and abroad, thus promoting the spread of China's excellent traditional culture in the world.

\section{INNOVATING NANYIN CULTURE PROMOTION STRATEGY}

\section{A. Continuously Promoting the Innovation of Nanyin Products}

Accelerating the deep integration of culture and science and technology is an effective way to promote the rapid development of culture, and is the driving force for promoting the multi-application of culture [4], which consists of two parts:

1) Innovation of Nanyin products: Establishing an excellent Nanyin culture is inseparable from the innovation of cultural content to enrich the cultural consumption connotation. Although the Nanyin art has profound connotations, it is difficult to attract the attention of modern society in both the form of performance and the music itself. Therefore, Nanyin culture is required to keep pace with the times and continue to innovate. Nanyin is mainly based on inheritance. Whether it is a civil society or a professional orchestra, the songs used in the performance are mostly original music. However, it is necessary to innovate while inheriting. In addition to the innovation of playing music, there can be some breakthroughs in the form of performance. Just like the combination of Peking Opera and popular music, Nanyin can also try to combine the blue-tone music to complement each other and provide more enlightenment for the innovation of Nanyin culture.

2) Increasing the integration of Nanyin culture and digital technology: Cultural innovation is inseparable from the integration of science and technology. The promotion of Nanyin culture needs to promote the integration of digital technology and Nanyin culture, further exerting the productivity of science and technology and culture and injecting strong impetus into the transformation of industrial structure and sustainable economic development. It is suggested to encourage to add modern scientific and technological elements to Nanyin's creation, so that Nanyin's works have a modern sense, which is more in line with the modern people's aesthetics, and is more favorable to the spread of Nanyin culture. At the same time, it is necessary to promote the mobile terminal application of Nanyin. On the one hand, people interested in Nanyin can understand and appreciate Nanyin more conveniently, and on the other hand, people can use technology to change the operation mode of Nanyin. For example, the information transformation mechanism of nanyin products is formulated to coordinate with software technology, platform operation and electronic product design to provide the Nanyin cultural products in line with the Times.

\section{B. Creating a Talent Training Mechanism to Promote Internet Plus Nanyin Culture Education}

After Nanyin was successfully selected as the "List of Representatives of Intangible Cultural Heritage of Humanity", the governments, society, schools, research organizations and other forces have joined forces to make 
great contributions to Nanyin's talent education. At present, Nanyin has entered the primary and secondary school subjects, secondary art colleges and colleges and universities, but there are still some shortcomings in the Nanyin talent training mechanism. For example, the faculty problem: the scientific research ability is not strong, and it is impossible to keep abreast of the latest information of the Nanyin discipline. Therefore, it is possible to innovate the talent training mechanism and promote the construction of the Nanyin education system against the Internet background.

The combination of Internet plus Nanyin culture will help to form a new ecosystem of innovative cultural industries. Internet plus Nanyin education refers to the use of mobile information technology and Internet platform to increase learning efficiency, to innovate and supplement in traditional teaching methods, such as the recent popular MOOC and flip classroom, which provide a reference for Nanyin education [5]. First of all, it is necessary to change the existing classroom teaching mode, although there are many primary and secondary schools to carry out the Nanyin course, the effect is not satisfactory. The use of Internet plus Nanyin teaching can fully mobilize the enthusiasm of Nanyin learners and Nanyin educators to ensure the effective teaching. Secondly, the Internet plus teaching model can reduce the teaching cost and realize the full sharing of online teaching resources. Thirdly, Internet plus Nanyin education helps to form a multi-dimensional learning space, break spatial and temporal limitations, and offer flexible teaching methods without affecting teaching quality. Finally, the use of Internet technology and the emergence of mobile learning tools have brought about the fragmentation of learning, providing the possibility for Nanyin learners to learn anytime and anywhere, which is beneficial to improve the efficiency of teaching to a certain extent.

\section{Internet Plus Database Model}

The digitization of Nanyin culture is not high, and many valuable materials cannot be inherited. In response to this situation, Internet technology can be used to build a systematic, complete and compatible Nanyin database. First of all, the old generation of artists will be visited to complete the collection of electronic data information such as video, recording and scores of first-hand materials. It is necessary to explore the history and culture of Nanyin through the use of other channels, collect and photography all kinds of ancient instruments and other supplies of Nanyin, as well as the important Nanyin music and singing methods that are popular among the people, and create various carriers including text, pictures, video and audio, etc., establish archives in a true, comprehensive and objective way, update data in time, and build a multi-level Nanyin database system that gathers collection, analysis, studies, teaching and describes [6]. The construction of the database will open up a broader space for the development of Nanyin culture, open the door for knowledge for Nanyin lovers and beginners, provide a variety of valuable reference materials for Nanyin, and pass on the memory of human beings. This is an important part of the spread of Nanyin culture.

\section{Internet Plus Nanyin Culture Brand Positioning and Promotion}

In the information age environment, the connection between the Internet and brand promotion is getting closer and closer. Therefore, it is necessary to re-interpret the construction and creation of the Nanyin brand in the Internet plus era with a new perspective and thinking mode, seize the opportunity and give full play to the convenience of Internet plus, do a good job in brand promotion to create an advantageous cultural brand.

Internet plus brand promotion refers to the use of information and communication technology and the Internet platform, the use of the means of emerging technology promotion to establish a solid image in the minds of consumers, stimulate and expand market demand, and open up potential markets, thereby increasing market share and enhancing brand value. Brand is the main focus of the development of cultural industry. The creation and promotion of Nanyin cultural industry brand is an important step in the promotion of Nanyin culture and cultural industry. The biggest advantage that Nanyin culture can use is its emotional value. Quanzhou Nanyin has a profound mass base, and various folk activities have the participation of Nanyin. At the same time, Nanyin is also popular in Hong Kong, Macao and Taiwan regions and Southeast Asian countries such as the Philippines, Indonesia, Singapore, Malaysia, Thailand, Myanmar, Vietnam, etc., and has become a spiritual bond to maintain Taiwan compatriots and overseas Chinese, and has also played an active role in increasing a sense of national identity. This advantage of Nanyin can be made full use of to give it a clear brand positioning — nostalgia.

In addition, there are new ways and means to develop and promote Nanyin: firstly, the spread of Nanyin's official website. Based on the official website of the Internet, the Nanyin culture and related content can be spread by means of text, images, audio, video, etc., and it is necessary to make full use of both lines of on-line advertisements on TV or the Internet, off-line sponsors of large-scale events, etc., to maximize the spread of Nanyin's official website; secondly, construction of the WeChat public account. Quanzhou Nanyin Culture can create an all-around communication and interactive public account of words, pictures and videos, which is also a powerful way for Nanyin to spread; thirdly, construction of official microblog. Nanyin can be used as a tourist card for Quanzhou. It is suggested that people take photos and write original articles and post them on microblog to attract the attention of many microblog fans to begin the tourism road of finding Nanyin culture; fourthly, shooting of public service advertisements. The biggest role of public service advertisements is to guide. Therefore, it is necessary to shoot the public announcements about Nanyin and implant announcements in some website forums and program videos to increase the dissemination and spread the charm of Nanyin. An excellent Nanyin public service advertisement can attract the audience's interest in Nanyin and expand the consumer group of Nanyin; fifthly, the spread of mobile client. People should focus on the spread of mobile platforms. For example, you can develop mobile 
phone software about Nanyin, add some entertainment information and learning curriculum theme, let more "mobile clan" subtly accept Nanyin, enjoy Nanyin anytime and anywhere, and let Nanyin enter the people's life circle.

\section{E. The Internet Plus Nanyin Cultural Industry Chain}

Nanyin culture is an intangible spiritual cultural resource. According to the cultural resource evaluation index system, Nanyin culture has very high historical value, aesthetic value, scientific education value, emotional value, direct economic value, indirect economic value, protection value and development value.

Therefore, Nanyin culture not only needs to establish a brand, form a Nanyin cultural industry chain, but also to form an interaction within the industry chain. For example, Nanyin Culture can form an industrial connection with arts and crafts, tourism experience and production technology. The Internet plus Nanyin cultural industry chain is to combine existing cultural development patterns, introduce information and communication technologies and Internet platforms, optimize existing industrial structures, broaden market channels, and form new production formats with Internet plus as the main driving force to create new development modes [7].

\section{CONCLUSION}

In short, the promotion of culture is of great significance to the implementation of China's cultural development strategy. Under this premise, it is necessary to actively seek the development, promotion and popularization of Nanyin culture and against the background of the "One Belt and One Road" strategy to formulate a suitable development strategy for this excellent cultural resource and provide reference for the development of more excellent historical culture.

\section{REFERENCES}

[1] Wang Yanxiu. Research on the Development of National Cultural Industry Based on Internet Age [J]. Yunnan Social Sciences, 2016(3): 185-188. (in Chinese)

[2] Wang Shan. Research on the Status Quo of Quanzhou Nanyin Inheritance [J]. Southeast Academic, 2011 (06): 307-312. (in Chinese)

[3] Wang Dandan. Reflections on the Inheritance of Nanyin. Chinese Musicology, 2014(01): 94-97. (in Chinese)

[4] Li Fengliang, Hu Penglin. Cultural and Technological Integration in the Internet Age 2014 General Report on Cultural Science and Technology Innovation [J]. Fujian Forum, 2015(12): 113-121. (in Chinese)

[5] Huang Meijiao. Changes and Challenges Brought by Internet Education to Higher Education [J]. Journal of Fujian Commercial College, 2016(1): 42-46. (in Chinese)

[6] Shang Jing. The Road to the Development of Nanyin in the Perspective of New Media [J]. News Front, 2015(05):135-136. (in Chinese)

[7] Huang Furong. Countermeasures and Model Innovation of Internet Culture Industry Development [J]. Statistics and Decision, 2015(24): 158-161. (in Chinese) 